\title{
REACTIVE HEMOPHAGOCYTIC LYMPHOHISTIOCYTOSIS IN SYSTEMIC LUPUS ERYTHEMATOSUS-A CASE REPORT AND LITERATURE REVIEW
}

\author{
TASNUVA SAIFUL ${ }^{1}$, QUADRAT-E- ELAHI ${ }^{1}$, MOSTAFIL KARIM $^{2}$, MAMUN MOSTAFI $^{3}$, ABDUL ALI MIA ${ }^{4}$
}

\begin{abstract}
Introduction: Hemophagocytic lymphohistiocytosis $(H L H)$ is a syndrome of immune overstimulation. HLH commonly manifests as multiple organ failure without apparent physiological stress. Primary $H L H$ relates to genetic defects, whereas secondary $H L H$ is triggered by infection, malignancy, or autoimmune disease. This paper describes the features of a young female patient with systemic lupus erythematous (SLE) who presented HLHS as the initial clinical manifestation.
\end{abstract}

Clinical observations: The patient had prolonged fever not associated to an infectious process and did not respond to broad-spectrum antibiotics.

\begin{abstract}
Discussion: The diagnosis of HLH secondary to SLE is complicated, because it has some features in common, but HLH is characterized by organomegaly, hyperferritinemia, hypofibrinogenemia, hypertriglyceridemia and cytopenia, a decrease in the erythrocyte sedimentation rate, unlike SLE. An eight week induction therapy with dexamethasone, etoposide, and intrathecal methotrexate increases survival in primary HLH and virus-associated secondary HLH. However, no standardized regimen exists for autoimmune associated HLH. Some patients respond to steroid monotherapy, while refractory patients may require cyclosporin, cyclophosphamide, or tacrolimus.
\end{abstract}

Conclusions: HS can be the initial manifestation of SLE and should be suspected in patients with organ enlargement, cytopenias, clotting disorders, liver disorders and prolonged fever unresponsive to antibiotics. Anakinra may be a treatment option in adult HS associated to SLE.

Keywords: Hemophagocytic Lymphohistiocytosis, Systemic Lupus Erythematosus, Autoimmune Disease.

Received: 20 December 2016

Accepted: 28 May 2017

\section{Introduction}

Hemophagocytic lymphohistiocytosis (HLH) is a disorder of immune dysregulation leading to systemic hyper inflammation due to uncontrolled proliferation of activated CD8 T cells, lymphocytes, and macrophages that secrete large amounts of inflammatory cytokines. ${ }^{1}$ It is a rare, life-threatening condition usually affecting children and young adults.

It comprises two different conditions; a primary or genetic and a secondary or acquired. These forms may be difficult to distinguish from each other. ${ }^{2-3}$ The primary is autosomal recessive form, also known as familial haemophagocytic lymphohistiocytosis (FHLH), is usually seen among children though adult cases have been reported. FHLH were first reported among in two siblings in 1952 and has an estimated incidence of 1 case per 50,000 live born children. Secondary (sHLH) or acquired HLH was first established as a distinct clinico-pathological entity by Risdall in 1979 18 which is more common among adults and typically occurs after strong immunological triggers (viral, bacterial, fungal, parasitic infections, collagenvascular diseases, and malignancies, particularly Tcell lymphomas). ${ }^{19-21}$

In regulated immune systems, natural killer (NK) cells attenuate histiocyte activity. Activation induced apoptosis also reduces the active $\mathrm{T}$ lymphocyte population. ${ }^{4}$ In $\mathrm{HLH}$, aberrant NK cell regulation or

1. Classified specialist in Medicine, $\mathrm{CMH}$, Dhaka

2. Classified specialist in Hematology, $\mathrm{CMH}$, Dhaka

3. Chief physician, $\mathrm{CMH}$, Dhaka

4. Consultant physician General, Bangladesh Armed Forces

Address of Correspondence: Major Dr. Tasnuva Saiful, FCPS (Medicine), Medicine specialist, CMH, Dhaka, Email: tasnuva.saiful@gmail.com

Bangladesh J Medicine 2017; 28 : 95-99 
absent activation induced apoptosis results in uncontrolled inflammation. The Histiocyte Society proposed a diagnostic criteria for HLH in 2004 (Table 2). ${ }^{5}$ Prominent features of HLH include fever, cytopenia, and acute organ failure ${ }^{5}$. HLH should be considered in patients with acute multiple organ failure without apparent physiological stress.

Delayed diagnosis is common because of its variable presentation. Affected patients often receive treatment for sepsis before their bone marrow examination identifies the underlying disorder. The diagnosis of secondary HLH is even more difficult because symptoms may mimic those of the triggering medical condition, such as SLE or viral infection.

Table-I

Classification of $\mathrm{HLH}$.

Genetic or primary HLH

- Familiar HLH

o Known genetic defects: for example, perforin or oth

o unknown genetic defects

- Immune deficiencies

o Chediak-Higashi

o Griscelli Syndrome

o X-linked lymphoproliferative syndrome

Acquired or secondary HLH

- Secondary to infections

- Secondary to endogenous products

- Secondary to rheumatic diseases

- Secondary to neoplasms

\section{Table-II}

Diagnostic criteria for $H L H$.

A. Molecular diagnosis: pathologic mutations of PRF1, UNC13D, Munc 18-2, Rab27a, STX11, SH2D1A, or BIRC4.

Or

B. Five of the eight criteria below are fulfilled:

1. Fever g" $38.5^{\circ} \mathrm{C}$

2. Splenomegaly

3. Cytopenia (at least 2 of 3 lineages in peripheral blood)

a. Hemoglobin $<9 \mathrm{~g} / \mathrm{dl}$

b. Platelet $<100000 / \mathrm{ml}$

c. Neutrophil $<1000 / \mathrm{ml}$

4. Hypertriglyceridemia (fasting level > $265 \mathrm{mg} / \mathrm{dl}$ ) and / or hypofibrinogenemia (< $150 \mathrm{mg} / \mathrm{dl}$ )

5. Hemophagocytosis in bone marrow, spleen, lymph nodes, or liver

6. Low or absent NK cell activity

7. Ferritin $>500 \mathrm{ng} / \mathrm{ml}$ (equivalent to $500 \mathrm{ug} / \mathrm{l}$ )

8. Elevated sCD25 (soluble IL-2 receptor.
We reported a case of $\mathrm{HLH}$ in a young patient with SLE in order to illustrate the spectrum of clinical features and to emphasis the importance of prompt diagnosis and initiation of therapy.

\section{Case report:}

A 14-year-old girl visited the emergency department with the complaints of generalized weakness with a recent history of high grade intermittent fever, polyarthralgia and oral ulcer for last 3 months. She had no history of headache, convulsion, vomiting, diarrhea, sore throat, cough, urinary complaints, trauma, documented hypoglycemia, recent change of medication or recent travel. She went to a local hospital where she was initially treated with Inj.Ceftriaoxone $2 \mathrm{gm} /$ day and received 02 unit blood transfusion due to low haemoglobin. She saw some improvement but continued presenting the symptoms as well as fever.

On admission, her body temperature was $103^{\circ} \mathrm{F}$, heart rate was $104 /$ minute, respiratory rate was $18 /$ minute, and blood pressure was 90/60 mmHg. Anemic conjunctiva was found. Discoid rash was distributed more in face, neck and trunk than limbs. Multiple lymph nodes of variable size and shape were palpable in cervical region. Abdominal palpation elicited hepatomegaly ( $\geq 3 \mathrm{~cm}$ from the right coastal margin) and splenomegaly ( $\geq 5 \mathrm{~cm}$ from the left costal margin). Chest survey revealed no abnormality. Neurologic examination was unremarkable. She received several courses of broad spectrum antibiotics (ceftriaxone, amoxicillin/clavulanic acid, levofloxacin), but didn't improved. Other associated infectious process was ruled out (Enteric fever, Malaria, kala-azar, TB, Lymphoproliferative disorder).

Her Laboratory investigations showed hemoglobin 7.4 $\mathrm{g} / \mathrm{dl}$, TLC $2000 / \mathrm{mm}^{3}$, and platelet count reduced to $30000 / \mathrm{mm}^{3}$. Total bilirubin was raised to $1.4 \mathrm{mg} / \mathrm{dl}$ (NR: 0.1-1.2 mg/dl) and indirect bilirubin $1 \mathrm{mg} / \mathrm{dl}$ (NR: 0-0.2 mg/dl). Direct and indirect Coombs tests were negative. Immunological screening was positive for ANA (1:160) with a homogenous pattern, anti-dsDNA was $134.1 \mathrm{IU} / \mathrm{ml}$ (normal: <18) and serum C3 and C4 complement factors were also low. The 24-hour urinary protein was 1.12 grams, but her other renal parameters like serum urea and creatinine were normal. She fulfilled seven of the American College of Rheumatology criteria for SLE, Including arthralgia, oral ulcer discoid rash, positive ANA, high titre of anti-dsDNA, leucopenia and thrombocytopenia, and proteinuria.

Screening tests done included Widal, HIV, Anti$\mathrm{HBsAg}$, Anti-HCV, dengue, brucella, EBV, and 
leptospira to detect an infectious cause and were all found to be negative. Her serum ferritin and fasting triglyceride was markedly elevated $1645 \mathrm{ug} / 1$ (NR: 20$290 \mathrm{ug} / 1$ ) and $505 \mathrm{mg} / \mathrm{dl}$ (normal < $150 \mathrm{mg} / \mathrm{dl}$ ) respectively. Her bone marrow shows hyper cellular marrow with normal plasma cells, lymphocytes and histiocytes. It revealed secondary reactive marrow due to peripheral consumption. No hemophagocytosis was found. She fulfilled five of the criteria for $\mathrm{HLH}$, including feverg" $38.5^{\circ} \mathrm{C}$, splenomegaly, Cytopenia, ferritin elevation and hypertriglyceridemia. The tests that were not met were Molecular diagnosis, quantifying natural killer cells, and sIL-2 receptor, tests that are not performed at our center.

During in-hospital course of illness, the patient received empirically injectable broad spectrum antibiotics (including meropenem) for febrile episodes and supportive treatment with blood transfusion, antipyretic and NSAIDs. She was started oral prednisolone $35 \mathrm{mg}$ / day (as per her body weight) and mycophenolate mofetil by consulting with nephrologist considering her primary diagnosis as SLE. The patient remained static during initial three days of therapy but subsequently her clinical and biochemical parameters improved with all continued supportive treatments. Serum ferritin concentration reflects disease activity and it decreases after steroid therapy. The patient became afebrile after 10 days of treatment. Size of liver and spleen decreased. Blood count improved as hemoglobin raised to 7.4 to $11.3 \mathrm{gm} / \mathrm{dl}$; TLC: 2.0 to $2.4 \mathrm{~mm}^{3}$, platelet 30 to $69 \times 10^{9} / 1$ ). Regarding HLH, she progressed well. The patient was discharged and sent home due to improvement. She is presently in remission and on regular follow-up at our outpatient department.

\section{Discussion:}

The prevalence of secondary HLH was estimated at $0.9 \%$ in patients with SLE. Autoimmune associated HLH commonly affects females with a median age of 43 years $^{6}$. The diagnosis of HLH secondary to SLE is complicated because they share some common characteristics. Lambotte et al. described 8 cases where HLS was diagnosed simultaneously with SLE in presence of pancytopenia, high ferritin and triglyceride level, which is highly suggestive of HS [5]. Parodi et al. proposed preliminary diagnostic criteria for macrophage activation syndrome as a complication of juvenile SLE. The diagnostic criteria have a sensitivity and specificity of $92.1 \%$ and $90.9 \%$, respectively ${ }^{14}$. This report describes an uncommon example of early-onset SLE in a young female. Macrophage activation syndrome (MAS) is a special form of HLH which occurs both in children and adults with autoimmune diseases ${ }^{9}$, MAS should be thought of as a form of HLH associated with a rheumatologic disease, rather than as a separate clinical entity and most commonly seen in association with systemic onset juvenile rheumatoid arthritis (sJRA) or adult onset Still's disease and rarely found with systemic lupus erythematosus or other entities ${ }^{10,11}$. It has been suggested by some rheumatologists that MAS be classified as a form of secondary $\mathrm{HLH}^{12,13}$. Table:3 shows the preliminary diagnostic criteria for macrophage activation syndrome as a complication of SLE proposed by Parodi et al. ${ }^{14}$

Table-III

Preliminary Diagnostic Criteria for Macrophage Activation Syndrome Complicating SLE.

\section{Clinical criteria}

a) Fever (>38 æ\%C)

b) Hepatomegaly ( $\geq 3 \mathrm{~cm}$ below the rib margin)

c) Splenomegaly ( $\geq 3 \mathrm{~cm}$ below the rib margin)

d) Hemorrhagic manifestations (purpura, easy bruising or bleeding gums)

e) Dysfunction of the central nervous system (irritability, disorientation, lethargy, headache, convulsions or coma)

Laboratory criteria

a) Cytopenia in 2 or more cell lines (TLC $<4 \times 109$ / 1 , hemoglobin $<90 \mathrm{~g} / 1$ or platelets $<150 \times 109 / 1)$

b) Increased aspartate aminotransferase $(>40$ units /1)

c) Increased lactate dehydrogenase(>567units/1)

d) Hypofibrinogenemia (fibrinogen $<1.5 \mathrm{~g} / 1$ )

e) Hypertriglyceridemia (triglycerides $>178 \mathrm{mg} / \mathrm{dl}$ )

f) Hyperferritinemia (ferritin $>500 \mathrm{~g} / \mathrm{l}$ )

\section{Histopathological criteria}

Evidence of hemophagocytic macrophages in bone marrow.

N.B.: For diagnosis, the simultaneous presence of at least one clinical criterion and at least 2 laboratory criteria is required. The bone marrow biopsy may be needed only in doubtful cases.

These criteria have some advantages and disadvantages. Advantages are the fact that a BM biopsy should be performed only in cases of diagnostic doubt, which seems reasonable because it is very invasive and often delays diagnosis and initiation of treatment. The most important disadvantage is that these criteria cannot distinguish from an infectious complication. All patients should have a bone marrow aspiration. However, frank haemophagocytosis may not be observed early in the course of the disease, 


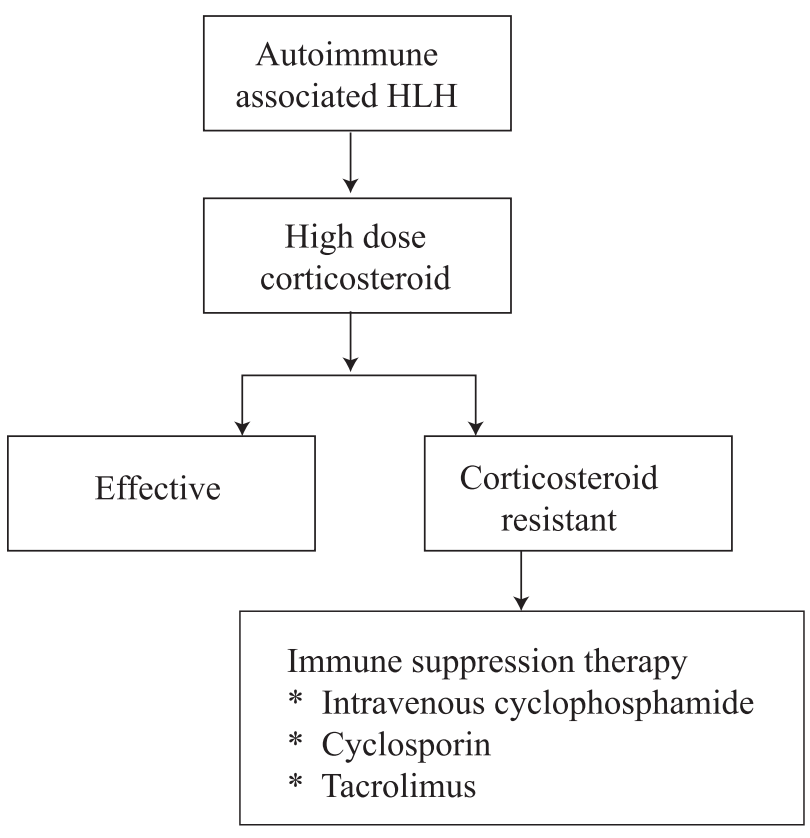

Fig.-1: Management of autoimmune associated $H L H$

and serial marrow aspirates may be helpful. ${ }^{15,16}$ Haemophagocytosis might be found in the first bone marrow aspiration of a FHLH patient but the absence of it will not rule out this diagnosis.

Certain laboratory parameters help us discern between HLH and active SLE. Thrombocytopenia is a better indicator HLH than leukopenia or anemia. Hyperferritinemia seems to have the strongest ability to discriminate between these two possibilities with a sensitivity and specificity of almost $100 \%$ patient resulting in secondary $\mathrm{HLH}$.

The current literature suggests that the goal of therapy for patients with HLH is to suppress lifethreatening inflammation by destroying immune cells. The first treatment protocol for HLH (HLH-94) consists of induction therapy with weekly treatments of dexamethasone and etoposide (VP-16), followed by cyclosporine. Intrathecal methotrexate is given to those with central nervous system disease. After induction, patients who are recovering are weaned off therapy, while those who are not improving are continued on therapy as a bridge to allogeneic hematopoietic cell transplantation. ${ }^{9}$ The newer HLH protocol initiated in 2004 (HLH-2004) differs from HLH-94 by earlier use of cyclosporine during the induction phase of treatment and adds hydrocortisone to intrathecal methotrexate. Treatment of secondary HLH is directed at control of the underlying condition. Steroids and other immune suppressants including Rituximab have also been reported in successfully treating HLH in the setting of SLE. ${ }^{17}$ Approximately
$56 \%$ of patients were resistant to steroid monotherapy 6 . Refractory cases may respond to cyclosporin, cyclophosphamide, or tacrolimus Figure-1.

In our patient, our treatment was targeted to control of SLE with nephropathy. So, we used high dose methylprednisolone followed by oral prednisolone and mycophenolate mofetil. Wong et al. reported an incidence of SLE-associated HS of 6 cases during a 3.5-year period among 250 SLE patients. They alluded to the fact that SLE-associated HLH might be underdiagnosed due to the overlap in clinical findings ${ }^{22}$. Because of the high mortality associated with HLH, the median survival being 02 months without treatment.

Survival rate increases to $55 \%$ after treatment ${ }^{8}$. Affected patients should receive treatment for the underlying rheumatologic disorders. Antimicrobial therapy is indicated to prevent severe bacterial infection during immune suppression therapy. Our patients responded to treatment, recovered and are presently on regular follow-up at the outpatient department.

HLS can be the initial clinical manifestation of SLE and should be suspected in patients with enlarged liver or spleen, cytopenias, clotting disorders, liver disorders and prolonged fever unresponsive to broadspectrum antibiotics, as occurred in our patient. Sometimes, the presenting features are so indistinct that, many cases may go unrecognized or be recorded as sepsis. Early diagnosis is very important for timely commencement of the treatment, before overwhelming disease activity makes irreversible damage and a response to treatment becomes less likely. SLE associated HLH portends poor prognosis. This case represents the importance of prompt diagnosis and treatment of such a potentially fatal clinical syndrome.

\section{Conclusion}

$\mathrm{HLH}$ is a life-threatening hyper-inflammatory syndrome which remains difficult to diagnose and can be easily overlooked or misdiagnosed. A prompt diagnosis is essential for treating HLS in the setting of SLE. Clinicians should raise a high index of suspicion in such a case.

\section{References}

1. Freeman HR, Ramanan AV. Review of haemophagocytic lymphohistiocytosis. Arch Dis Child. 2011; 92. Henter J-I, Arico M, Elinder G, et al. Familial

2. Haemophagocytic lymphohistiocytosis (primary HLH). Hematol Oncol Clin North Am 1998;12: 417-433. 
3. Janka G, Elinder G, Imashuku S, et al. Infectionand malignancy associated haemophagocytic syndromes:Secondary haemophagocytic lymphohistiocytosis. Hematol Oncol Clin North Am 1998;12: 435-444.6(7):688-693.

4. Filipovich A, McClain K, Grom A. Histiocytic Disorders: Recent Insights into Pathophysiology and Practical Guidelines. Biol Blood Marrow Transplant. 2010; 16(1 suppl): S82-S89.

5. Henter JI, Horne A, Arico M, Egeler RM, Filipovich AH, Imashuku S, et al. HLH-2004: Diagnostic and therapeutic guidelines for hemophagocytic lymphohistiocytosis. Pediatr Blood Cancer. 2007; 48(2): 124-131.

6. Fukaya S, Yasuda S, Hashimoto T, Oku K, Kataoka $\mathrm{H}$, Horita $\mathrm{T}$, et al. Clinical features of haemophagocytic syndrome in patients with systemic autoimmune diseases: analysis of 30cases. Rheumatology (Oxford). 2008; 47 (11): 1686-1691.

7. Horne A, Trottestam H, Arico M, Egeler RM, Filipovich AH,Gadner H, et al. Frequency and spectrum of central nervous system involvement in 193 children with haemophagocytic lymphohistiocytosis. Br J Haematol. 2008; 140(3): 327-335.

8. Henter JI, Samuelsson-Horne A, Arico M, Egeler RM,Elinder G, Filipovich AH, et al. T Haemophagocytic lymphohistiocytosis with HLH94immunochemotherapy and bone marrow transplantation.Blood. 2002; 100 (7): 2367-2373.

9. Janka GE. Haemophagocytic Syndromes. Blood Rev 2007; 21: 245-53.

10. Stephan J1, Kone-Paut I, Galambrun C, Mouy R, Bader-Meunier B, Prieur AM. Reactive haemophagocytic syndrome in children with inflammatory disorders. Aretrospective study of 24 patients. Rheumatology 2001;40:1285-92.

11. Ravelli A. Macrophage activation syndrome. Curr Opin Rheumatol 2002;14:548-52.

12. Ramanan AV, Schneider R. Macrophage activation syndrome- what's in a name! J Rheumatol 2003;30:2513-6.
13. Athreya BH. Is macrophage activation syndrome a newentity? Clin Exp Rheumatol 2002;20:121-3.

14. 5. Parodi A, Davì S, Pringe $A B$, Pistorio A, Ruperto $\mathrm{N}$, Magni-Manzoni S, et al., Lupus Working Group of the Paediatric Rheumatology European Society. Macrophage activation syndrome in juvenile systemic lupus erythematosus: a multinational multicenter study of thirty-eight patients. Arthritis Rheum. 2009;60:3388-99.

15. Gupta A, Weitzman S, Abdelhaleem M. The role of haemophagocytosis in bone marrow aspirates in the diagnosis of haemophagocytic lymphohistocytosis. Pediatr Blood Cancer 2008 Feb; 50: 192-194.

16. Gupta A, Tyrrell P, Valani R, Benseler S, Weitzman S, Abdelhaleem M. Pediatr Blood Cancer 2008; 51:402-404.

17. Hochberg MC. Updating the American College of Rheumatology revised criteria for the classification of systemic lupus erythematosus. Arthritis Rheum.1997; 40:1725 [letter].

18. Risdall RJ, McKenna RW, Nesbit ME, et al. Virus associated haemophagocytic syndrome: a benign histiocytic proliferation distinct from malignant histiocytosis. Cancer 1979;44: 993-1002

19. Chang CS, Wang CH, Su I J , Chen YC, Shen MC. Hematophagic histiocytosis clinicopathologic analysis of 23 cases with special reference to the association with peripheral T-cell lymphoma. J Formos Med Assoc 1994;93:421-8.

20. Kadin ME, Kamoun M, Lamberg J. Erythrophagocytic $\mathrm{T}$ gamma lymphoma: a clinicopathologic entity resembling malignant histiocytosis. N Engl J Med 1981;304:648-53.

21. Yao M, Cheng A, Su I. Clinicopathological spectrum of haemophagocytic syndrome in Epstein-Barr virusassociated peripheral T-cell lymphoma. $\mathrm{Br} \mathrm{J}$ Haematol 1994;87:535-43.

22. K.-F. Wong, P.-K. Hui, J. K. C. Chan, Y.-W. Chan, and S.-Y. Ha, "The acute lupus hemophagocytic syndrome," Annals of Internal Medicine, vol. 114, no. 5, pp. 387-390, 1991. 